\title{
Constrained Vector-Valued Dynamic Game and Symmetric Duality for Multiobjective Variational Problems
}

\author{
I. Husain ${ }^{*}$ and Vikas K. Jain ${ }^{*}$ \\ Department of Mathematics, Jaypee University of Engineering and Technology, Guna (M.P), India
}

\begin{abstract}
A certain constrained vector-valued dynamic game is formulated and shown to be equivalent to a pair of multiobjective symmetric dual variational problems which have more general formulations than those studied earlier. A number of duality theorems, are established under suitable generalized convexity assumptions on the functionals. Selfduality reflecting symmetric dynamic games is investigated. The constrained vector-valued dynamic game is also regarded as equivalent to a pair of symmetric multiobjective dual variational problems with natural boundary conditions rather than fixed end points. Finally, it is indicated that our results can be considered as dynamic generalizations of those already existing in the literature.
\end{abstract}

AMS-Mathematics Subject Classification: Primary 90C30, Secondary 90C11, 90C20, 90C26.

Keywords: Vector-valued dynamic games, generalized convexity, multiobjective dynamic games, multiobjective variational problems, selfduality, symmetric duality.

\section{INTRODUCTION}

The applications of game-theoretic ideas are quite extensive and have recently been enjoying increased popularity in various human activities, as it provides a new perspective on optimization, networking and control problems. So the search for elegant methods for solving a general strategic game is very natural. Charnes [1] established that every matrix game is equivalent to linear programming. The results in [1] imply that every two-person, zero-sum can be solved by simplex method of linear programming. The advantage of linear programming techniques is that it provides solution to a mixed strategy game of any size. Motivated with this observation, many researchers studied equivalence between a scalar-valued game and a certain mathematical programming problem. Cottle [2] was the first to establish the equivalence between an unconstrained game having a nonlinear convex-concave payoff function and the corresponding symmetric dual programming problems. Since then several authors, notably, Chandra et al. [3] Corley [4] and Prasad and Sreenivas [5] extended partially the results of constrained scalar-valued game to a certain vector-valued game. Later Mond et al. [6] extended the results of Kawaguchi and Maruyama [7] to the nonlinear setting and proved that a constrained game is equivalent to a pair of Symmetric dual nonlinear programming problems, appearing similar to those of Mond-Weir [8].

The dynamic games are basically concerned with the modeling of large scale systems which have independent

*Address correspondence to these authors at the Department of Mathematics, Jaypee University of Engineering and Technology, Guna (M.P), India; Tel: +918305835387; Fax: +91-7544-267011;

E-mails: ihusain11@yahoo.com,jainvikas13@yahoo.com decision makers with individual payoff (or reward) functions. Applications of dynamic games can be experienced in solving some important problems relating to environment resources, aerospace and energy managements. So their domain of applications is naturally wider than those of static games. In order to extend the results of Mond et al. [6] to dynamic setting involving variational problems, recently Husain and Ahmad [9] have constructed a scalarvalued game and shown it to be equivalent to pair of symmetric dual variational problems general formulations whose formulations are more general then that of [10]. They established various duality results under convexity and generalized convexity assumptions on the appropriate functionals.

The purpose of this research is to extend the results of Husain and Ahmad [9] to multiobjective setting by formulating a constrained vector-valued dynamic game and established its equivalence to a pair of multiobjective symmetric dual variational problems. For this pair of variational problems, duality results are derived under suitable generalized convexity hypotheses. A pair of selfdual multiobjective variational problems reflecting its equivalence to symmetric multiobjective dynamic game, is formulated. Finally game equivalent problems with natural boundary values are constructed and their relationship with their static counterparts [11] is indicated. In order to understand the multiobjective setting of dynamic game one may be referred to go through "Zhukovskii and Salukvadze" [12].

\section{FORMULATION OF VECTOR-VALUED DYNAMIC GAMES}

In a game, if the process is controlled by p-players whose interested suffer from conflicts, then the objectives of an individual player cannot be expressed in terms of one index 
and hence results into multiobjective functions. Such specific characteristics constitute vector-valued game problems where each player wishes to optimize his own multiple criteria (pay- off function) or games with two player of which one wishes to maximize and the other wishes to minimize the same vector function. These situations provide motivation to study vector-valued constrained dynamic games.

To establish the equivalence of a constrained vectorvalued dynamic game to a pair of symmetric variational problems, we associate the vector-valued game

$G=(X, Y, F(x, y))$ to the game $G^{\prime}=\left(X, Y, \lambda^{T} F(x, y)\right)$, where

$$
\begin{aligned}
& X=\left\{\begin{array}{l}
x: I \rightarrow R^{n} \mid x(a)=0, x(b)=0, p_{i}(t, x, \dot{x}) \geq 0, \\
t \in I, i=1,2, \ldots ., k \text { and } I=[a, b] \subset R
\end{array}\right\} \text { and } \\
& Y=\left\{\begin{array}{l}
y: I \rightarrow R^{m} \mid y(a)=0, y(b)=0, q_{j}(t, y, \dot{y}) \geqq 0, t \in I, \\
j=1,2, \ldots ., l
\end{array}\right\}
\end{aligned}
$$

with

$$
\begin{aligned}
& p_{i}: I \times R^{n} \times R^{n} \rightarrow R, i=1,2, \ldots ., k \\
& q_{j}: I \times R^{n} \times R^{n} \rightarrow R, j=1,2, \ldots, l
\end{aligned}
$$

have continuous derivative up to and including second order with respect to each of their arguments $\mathrm{x}$ and $\mathrm{y}$ and $x: I \rightarrow R^{n}, y: I \rightarrow R^{m}$ with derivatives $\dot{x}$ and $\dot{y}$ with respect to t.

$$
\begin{aligned}
& F: X \times Y \rightarrow R^{p}, \text { defined by } \\
& F(x, y)=\left(\begin{array}{l}
\int_{a}^{b} f^{1}(t, x, \dot{x}, y, \dot{y}) d t, \ldots . ., \\
b \\
\int_{a}^{b} f^{p}(t, x, \dot{x}, y, \dot{y}) d t
\end{array}\right),
\end{aligned}
$$

where $f^{i}: I \times R^{n} \times R^{n} \times R^{m} \times R^{m} \rightarrow R$ is assumed to be continuously differentiable function $f, i=1,2, \ldots, p$

(iv) $\mathrm{X}$ and $\mathrm{Y}$ represent the strategy spaces for the players I and II respectively and $\lambda^{T} F(x, y)$ with $\lambda=\left(\lambda^{1}, \ldots \ldots . ., \lambda^{p}\right) \in R^{p}$ represents the payoff to the player II from the player I. The player I is assumed to be a minimizing player and player II a maximizing player. Thus the player I wishes to solve $\min _{x \in X} \max _{y \in Y} \lambda^{T} F(x, y)$ and the player II wishes to solve $\max _{y \in Y} \min _{x \in X} \lambda^{T} F(x, y)$.

This is to be noted here that throughout the analysis of this research, the following convention for the inequalities will be used:
If $\alpha, \beta \in R^{n}$ with

$\alpha=\left(\alpha^{1}, \alpha^{2}, \ldots, \alpha^{n}\right)$ and $\beta=\left(\beta^{1}, \beta^{2}, \ldots, \beta^{n}\right)$, then

$\alpha \geqq \beta \Leftrightarrow \alpha^{\mathrm{i}} \geqq \beta^{i},(i=1,2, \ldots, n)$

$\alpha \geq \beta \Leftrightarrow \alpha \geqq \beta$ and $\alpha \neq \beta$

$\alpha>\beta \Leftrightarrow \alpha^{\mathrm{i}}>\beta^{i},(i=1,2, \ldots, n)$.

Now $\min _{x \in X} \max _{y \in Y} \lambda^{T} F(x, y)$ and $\max _{y \in Y} \min _{x \in X} \lambda^{T} F(x, y)$ are equivalent to the following problem $(\mathrm{P})$ and(D):

(P): $\quad$ Minimize $\int_{a}^{b} \lambda^{T} F(t, x, \dot{x}, y, \dot{y}) d t$

subject to

$$
\begin{aligned}
& x(a)=0=x(b) \\
& y(a)=0=y(b) \\
& \left(\begin{array}{l}
\lambda^{T} f_{y}(t, x, \dot{x}, y, \dot{y})-\mu(t)^{T} q_{y}(t, y, \dot{y})- \\
D\left(\lambda^{T} f_{\dot{y}}(t, x, \dot{x}, y, \dot{y})-\mu(t)^{T} q_{\dot{y}}(t, y, \dot{y})\right)
\end{array}\right) \leqq 0, t \in I \\
& \int_{a}^{b} y(t)^{T}\left[\left(\begin{array}{l}
\lambda^{T} f_{y}(t, x, \dot{x}, y, \dot{y})-\mu(t)^{T} q_{y}(t, y, \dot{y})- \\
D\left(\lambda^{T} f_{\dot{y}}(t, x, \dot{x}, y, \dot{y})-\mu(t)^{T} q_{\dot{y}}(t, y, \dot{y})\right)
\end{array}\right)\right] d t \geqq 0 \\
& \int_{a}^{b} \mu(t)^{T} q(t, y, \dot{y}) d t \geqq 0 \\
& p(t, x, \dot{x}) \geqq 0, t \in I \\
& x(t) \geqq 0, \mu(t) \geqq 0, t \in I, \\
& \lambda>0 . \\
& \text { (D): Maximize } \int_{a}^{b} \lambda^{T} F(t, u, \dot{u}, v, \dot{v}) d t
\end{aligned}
$$

subject to

$$
\begin{aligned}
& u(a)=0=u(b) \\
& v(a)=0=v(b)
\end{aligned}
$$$$
\left(\begin{array}{l}
\lambda^{T} f_{u}(t, u, \dot{u}, v, \dot{v})-\gamma(t)^{T} p_{u}(t, u, \dot{u})- \\
D\left(\lambda^{T} f_{\dot{u}}(t, u, \dot{u}, v, \dot{v})-\gamma(t)^{T} p_{\dot{u}}(t, u, \dot{u})\right)
\end{array}\right) \geqq 0, t \in I
$$$$
\int_{a}^{b} u(t)^{T}\left[\left(\begin{array}{l}
\lambda^{T} f_{u}(t, u, \dot{u}, v, \dot{v})-\gamma(t)^{T} p_{u}(t, u, \dot{u})- \\
D\left(\lambda^{T} f_{\dot{u}}(t, u, \dot{u}, v, \dot{v})-\gamma(t)^{T} p_{\dot{u}}(t, u, \dot{u})\right)
\end{array}\right)\right] d t \leqq 0
$$$$
\int_{a}^{b} \gamma(t)^{T} p(t, u, \dot{u}) d t \leqq 0
$$

$q(t, v, \dot{v}) \leqq 0, t \in I$

$v(t) \geqq 0, \gamma(t) \geqq 0, t \in I$, 
$\lambda>0$.

Corresponding to the above variational problems $(\mathrm{P})$ and (D), we have the following multiobjective variational problems:

(VP): Minimize $\left(\int_{a}^{b} f^{1}(t, x, \dot{x}, y, \dot{y}) d t, \ldots, \int_{a}^{b} f^{p}(t, x, \dot{x}, y, \dot{y}) d t\right)$ subject to

$$
\begin{aligned}
& x(a)=0=x(b) \\
& y(a)=0=y(b) \\
& \left(\begin{array}{l}
\lambda^{T} f_{y}(t, x, \dot{x}, y, \dot{y})-\mu(t)^{T} q_{y}(t, y, \dot{y})- \\
D\left(\lambda^{T} f_{\dot{y}}(t, x, \dot{x}, y, \dot{y})-\mu(t)^{T} q_{\dot{y}}(t, y, \dot{y})\right)
\end{array}\right) \leqq 0, t \in I \\
& \int_{a}^{b} y(t)^{T}\left[\left(\begin{array}{l}
\lambda^{T} f_{y}(t, x, \dot{x}, y, \dot{y})-\mu(t)^{T} q_{y}(t, u, \dot{u})- \\
D\left(\lambda^{T} f_{\dot{y}}(t, x, \dot{x}, y, \dot{y})-\mu(t)^{T} q_{\dot{y}}(t, u, \dot{u})\right)
\end{array}\right)\right] d t \geqq 0 \\
& \int_{a}^{b} \mu(t)^{T} q(t, y, \dot{y}) d t \geqq 0 \\
& p(t, x, \dot{x}) \geqq 0, t \in I \\
& x(t) \geqq 0, \mu(t) \geqq 0, t \in I \\
& \lambda>0
\end{aligned}
$$

(VD): Maximize

$$
\left(\int_{a}^{b} f^{1}(t, u, \dot{u}, v, \dot{v}) d t, \ldots \ldots ., \int_{a}^{b} f^{p}(t, u, \dot{u}, v, \dot{v}) d t\right)
$$

subject to

$$
\begin{aligned}
& u(a)=0=u(b) \\
& v(a)=0=v(b) \\
& \left(\begin{array}{l}
\lambda^{T} f_{u}(t, u, \dot{u}, v, \dot{v})-\gamma(t)^{T} p_{u}(t, u, \dot{u})- \\
D\left(\lambda^{T} f_{\dot{u}}(t, u, \dot{u}, v, \dot{v})-\gamma(t)^{T} p_{\dot{u}}(t, u, \dot{u})\right)
\end{array}\right) \geqq 0, t \in I \\
& \int_{a}^{b} u(t)^{T}\left[\left(\begin{array}{l}
\lambda^{T} f_{u}(t, u, \dot{u}, v, \dot{v})-\gamma(t)^{T} p_{u}(t, u, \dot{u})- \\
D\left(\lambda^{T} f_{\dot{u}}(t, u, \dot{u}, v, \dot{v})-\gamma(t)^{T} p_{\dot{u}}(t, u, \dot{u})\right)
\end{array}\right)\right] d t \leqq 0 \\
& \int_{a}^{b} \gamma(t)^{T} p(t, u, \dot{u}) d t \leqq 0 \\
& q(t, v, \dot{v}) \leqq 0, t \in I \\
& v(t) \geqq 0, \gamma(t) \geqq 0, t \in I \\
& \lambda>0
\end{aligned}
$$

\section{MULTIOBJECTIVE SECOND-ORDER SYMMETRIC DUALITY}

In this section, we shall prove duality relationship between the problems (VP) and (VD). For this following definitions are needed:

Consider the following multiobjective variational problem studied by Bector and Husain [13]:

$(\widehat{P})$ Minimize

$\left(\int_{a}^{b} \phi^{1}(t, x(t), \dot{x}(t)) d t, \ldots \ldots . . \int_{a}^{b} \phi^{p}(t, x(t), \dot{x}(t)) d t\right)$

subject to

$$
\begin{aligned}
& x(a)=\alpha, x(b)=\beta \\
& g(t, x(t), \dot{x}(t)) \leq 0, t \in I .
\end{aligned}
$$

Let $\mathrm{L}$ denote the set of all feasible solutions of $(\widehat{P})$, i.e.

$L=\left\{x \in C\left(I, R^{n}\right): x(a)=\alpha, x(b)=\beta, g(t, x(t), \dot{x}(t)) \leq 0, t \in I\right\}$, where $C\left(I, R^{n}\right)$ denote the space of piecewise smooth functions $\mathrm{x}$ with norm $\|x\|=\|x\|_{\infty}+\|D x\|_{\infty}$, where the differentiation operator $\mathrm{D}$ is given by $u=D s \Leftrightarrow x(t)=\alpha+\int_{0}^{t} u(s) d s$ where $\alpha$ is a given boundary value. Therefore $\frac{d}{d t} \equiv D$ except at discontinuities.

Definition 1. A point $x^{0} \in L$ is said to be an efficient solution of $(\widehat{P})$ if for all $x \in L$,

$\int_{a}^{b} \phi^{i}\left(t, x^{0}(t), \dot{x}^{0}(t)\right) d t \geq \int_{a}^{b} \phi^{i}(t, x(t), \dot{x}(t)) d t$ for all

$i \in\{1,2, \ldots, p\}$

$\Rightarrow \int_{a}^{b} \phi^{i}\left(t, x^{0}(t), \dot{x}^{0}(t)\right) d t=\int_{a}^{b} \phi^{i}(t, x(t), \dot{x}(t)) d t$ for all

$i \in\{1,2, \ldots, p\}$.

The point $x^{o}$ is said to be a properly efficient solution of $(\widehat{P})$, if there exists a scalar $\mathrm{M}>0$ such that, for all $i \in\{1,2, \ldots, p\}$,

$\int_{a}^{b} \phi^{i}\left(t, x^{0}(t), \dot{x}^{0}(t)\right) d t-\int_{a}^{b} \phi^{i}(t, x(t), \dot{x}(t)) d t$

$\leq M\left(\int_{a}^{b} \phi^{j}(t, x(t), \dot{x}(t)) d t-\int_{a}^{b} \phi^{j}\left(t, x^{0}(t), \dot{x}^{0}(t)\right) d t\right)$,

for some $\mathrm{j}$, such that

$\left(\int_{a}^{b} \phi^{j}(t, x(t), \dot{x}(t)) d t>\int_{a}^{b} \phi^{j}\left(t, x^{0}(t), \dot{x}^{0}(t)\right) d t\right)$ 
whenever $x \in X$, and an $i \in\{1,2, \ldots, p\}$ such that

$$
\left(\int_{a}^{b} \phi^{i}(t, x(t), \dot{x}(t)) d t>\int_{a}^{b} \phi^{i}\left(t, x^{0}(t), \dot{x}^{0}(t)\right) d t\right) .
$$

An efficient solution that is not properly efficient is said to be improperly efficient. Thus for $x^{0}$ to be improperly efficient means that to every sufficiently large $M>0$, there is an

$$
\left(\int_{a}^{b} \phi^{i}(t, x(t), \dot{x}(t)) d t>\int_{a}^{b} \phi^{i}\left(t, x^{0}(t), \dot{x}^{0}(t)\right) d t\right)
$$

and

$$
\begin{aligned}
& \int_{a}^{b} \phi^{i}\left(t, x^{0}(t), \dot{x}^{0}(t)\right) d t-\int_{a}^{b} \phi^{i}(t, x(t), \dot{x}(t)) d t \\
& >M\left(\int_{a}^{b} \phi^{j}(t, x(t), \dot{x}(t)) d t-\int_{a}^{b} \phi^{j}\left(t, x^{0}(t), \dot{x}^{0}(t)\right) d t\right) .
\end{aligned}
$$

for all $i \in\{1,2, \ldots, p\}$

satisfying $\int_{a}^{b} \phi^{j}(t, x(t), \dot{x}(t)) d t>\int_{a}^{b} \phi^{j}\left(t, x^{0}(t), \dot{x}^{0}(t)\right) d t$.

Definition 2. A point $x^{0} \in L$ is said to be an weak minimum for $(\hat{P})$ if there exists no other $x \in L$ for which

$$
\int_{a}^{b} \phi\left(t, x^{0}(t), \dot{x}^{0}(t)\right) d t>\int_{a}^{b} \phi(t, x(t), \dot{x}(t)) d t
$$

From this, it follows that if $x^{0} \in L$ is efficient $(\widehat{P})$, then it is also a weak minimum for $(\widehat{P})$.

We shall use $\mathrm{F}$ and $\mathrm{G}$ for the sets of feasible solutions for the primal and dual multiobjective variational problems (VP) and (VD) respectively.

Theorem 1. (Weak Duality). Let

$\left(\mathrm{A}_{1}\right):(x(t), y(t), \mu(t), \lambda) \in F$ and $(u(t), v(t), \gamma(t), \lambda) \in G$,

$\left(\mathrm{A}_{2}\right): \int_{a}^{b}\left\{\lambda^{T} f(t, ., ., y(t), \dot{y}(t))-\gamma(t)^{T} p(t, .,).\right\} d t$

be pseudo-convex in $(x, \dot{x})$, and

$\left(\mathrm{A}_{3}\right): \int_{a}^{b}\left\{\lambda^{T} f(t, x(t), \dot{x}(t), . .)-.\mu(t)^{T} q(t, .,).\right\} d t$

be pseudo-convex in $(y, \dot{y})$

Then

$$
\int_{a}^{b} f(t, x(t), \dot{x}(t), y(t), \dot{y}(t)) d t \leq \int_{a}^{b} f(t, u(t), \dot{u}(t), v(t), \dot{v}(t)) d t .
$$

Proof: For $(x(t), y(t), \mu(t), \lambda) \in F$

and

$(u(t), v(t), \gamma(t), \lambda) \in G$,

inequalities (7), (11) and (12),

$0 \leq \int_{a}^{b}(x(t)-u(t))^{T}\left\{\begin{array}{l}\lambda^{T} f_{u}(t, u(t), \dot{u}(t), v(t), \dot{v}(t))- \\ \gamma(t)^{T} p_{u}(t, u(t), \dot{u}(t)) \\ -D\left(\begin{array}{l}\lambda^{T} f_{\dot{u}}(t, u(t), \dot{u}(t), v(t), \dot{v}(t))- \\ \gamma(t)^{T} p_{\dot{u}}(t, u(t), \dot{u}(t))\end{array}\right.\end{array}\right\} d t$

$=\int_{a}^{b}\left[\begin{array}{c}(x(t)-u(t))^{T}\left\{\begin{array}{l}\lambda^{T} f_{u}(t, u(t), \dot{u}(t), v(t), \dot{v}(t))- \\ \gamma(t)^{T} p_{u}(t, u(t), \dot{u}(t))\end{array}\right\} \\ +(\dot{x}(t)-\dot{u}(t))^{T}\left\{\begin{array}{l}\lambda^{T} f_{\dot{u}}(t, u(t), \dot{u}(t), v(t), \dot{v}(t))- \\ \gamma(t)^{T} p_{\dot{u}}(t, u(t), \dot{u}(t))\end{array}\right\}\end{array}\right] d t$

$-\left.(x(t)-u(t))^{T}\left\{\begin{array}{l}\lambda^{T} f_{\dot{u}}(t, u(t), \dot{u}(t), v(t), \dot{v}(t))- \\ \gamma(t)^{T} p_{\dot{u}}(t, u(t), \dot{u}(t))\end{array}\right\}\right|_{t=a} ^{t=b}$

Using (1) and (9), we have

$\int_{a}^{b}\left[\begin{array}{l}(x(t)-u(t))^{T}\left\{\begin{array}{l}\lambda^{T} f_{u}(t, u(t), \dot{u}(t), v(t), \dot{v}(t))- \\ \gamma(t)^{T} p_{u}(t, u(t), \dot{u}(t))\end{array}\right\} \\ +(\dot{x}(t)-\dot{u}(t))^{T}\left\{\begin{array}{l}\lambda^{T} f_{\dot{u}}(t, u(t), \dot{u}(t), v(t), \dot{v}(t))- \\ \gamma(t)^{T} p_{\dot{u}}(t, u(t), \dot{u}(t))\end{array}\right\}\end{array}\right] d t \geqq 0$

which, because of $\left(A_{1}\right)$ yields

$\int_{a}^{b}\left(\lambda^{T} f(t, x(t), \dot{x}(t), v(t), \dot{v}(t))-\gamma(t)^{T} p_{u}(t, x(t), \dot{x}(t))\right) d t$
$\geqq \int_{a}^{b}\left(\lambda^{T} f(t, u(t), \dot{u}(t), v(t), \dot{v}(t))-\gamma(t)^{T} p_{\dot{u}}(t, u(t), \dot{u}(t))\right) d t$

In view of

$\int_{a}^{b} \gamma(t)^{T} p_{u}(t, x(t), \dot{x}(t)) d t \geqq 0, t \in I$

and

$$
\int_{a}^{b} \gamma(t)^{T} p_{\dot{u}}(t, u(t), \dot{u}(t)) d t \geqq 0,
$$

this inequality implies

$$
\begin{aligned}
& \int_{a}^{b}\left(\lambda^{T} f(t, x(t), \dot{x}(t), v(t), \dot{v}(t))\right) d t \geqq \\
& \int_{a}^{b}\left(\lambda^{T} f(t, u(t), \dot{u}(t), v(t), \dot{v}(t))\right) d t
\end{aligned}
$$

From (3), (4) and (15), we have 


$$
\begin{aligned}
& 0 \geq \int_{a}^{b}(v(t)-y(t))^{T}\left\{\begin{array}{l}
\lambda^{T} f_{y}(t, x(t), \dot{x}(t), y(t), \dot{y}(t))- \\
\mu(t)^{T} q_{y}(t, y(t), \dot{y}(t)) \\
-D\left(\begin{array}{l}
\lambda^{T} f_{\dot{y}}(t, x(t), \dot{x}(t), y(t), \dot{y}(t))- \\
\mu(t)^{T} q_{\dot{y}}(t, y(t), \dot{y}(t))
\end{array}\right)
\end{array}\right\} d t \\
& =\int_{a}^{b}\left[\begin{array}{l}
(v(t)-y(t))^{T}\left\{\begin{array}{l}
\lambda^{T} f_{y}(t, x(t), \dot{x}(t), y(t), \dot{y}(t))- \\
\mu(t)^{T} q_{y}(t, y(t), \dot{y}(t))
\end{array}\right\} \\
+(\dot{v}(t)-\dot{y}(t))^{T}\left\{\begin{array}{l}
\lambda^{T} f_{\dot{y}}(t, x(t), \dot{x}(t), y(t), \dot{y}(t))- \\
\mu(t)^{T} q_{\dot{y}}(t, y(t), \dot{y}(t))
\end{array}\right\}
\end{array}\right] d t \\
& -\left[\begin{array}{l}
\lambda^{T} f_{\dot{y}}(t, x(t), \dot{x}(t), y(t), \dot{y}(t))- \\
\mu(t)^{T} q_{\dot{y}}(t, y(t), \dot{y}(t))
\end{array}\right]_{t=a}^{t=b}
\end{aligned}
$$

(By integration by parts)

Using (2) and (10), we have

$$
=\int_{a}^{b}\left[\begin{array}{c}
(v(t)-y(t))^{T}\left\{\begin{array}{l}
\lambda^{T} f_{y}(t, x(t), \dot{x}(t), y(t), \dot{y}(t))- \\
\mu(t)^{T} q_{y}(t, y(t), \dot{y}(t))
\end{array}\right\} \\
+(\dot{v}(t)-\dot{y}(t))^{T}\left\{\begin{array}{l}
\lambda^{T} f_{\dot{y}}(t, x(t), \dot{x}(t), y(t), \dot{y}(t))- \\
\mu(t)^{T} q_{\dot{y}}(t, y(t), \dot{y}(t))
\end{array}\right\}
\end{array}\right] d t \leq 0
$$

By pseudoconcavity of

$\int_{a}^{b}\left\{\lambda^{T} f(t, x(t), \dot{x}(t), . .)-.\mu(t)^{T} q(t, . .).\right\} d t$

this yields,

$$
\begin{aligned}
& \int_{a}^{b}\left\{\lambda^{T} f(t, x(t), \dot{x}(t), v(t), \dot{v}(t))-\mu(t)^{T} q(t, v(t), \dot{v}(t))\right\} d t \\
& \leq \int_{a}^{b}\left\{\lambda^{T} f(t, x(t), \dot{x}(t), y(t), \dot{y}(t))-\mu(t)^{T} q(t, y(t), \dot{y}(t))\right\} d t
\end{aligned}
$$

which, in view of

$$
\int_{a}^{b} \mu(t)^{T} q(t, y(t), \dot{y}(t)) d t \geqq 0
$$

and

$$
-\int_{a}^{b} \mu(t)^{T} q(t, v(t), \dot{v}(t)) d t \geqq 0 \text { implies }
$$$$
\begin{aligned}
& \int_{a}^{b}\left\{\lambda^{T} f(t, x(t), \dot{x}(t), v(t), \dot{v}(t))\right\} d t \leqq \\
& \int_{a}^{b}\left\{\lambda^{T} f(t, x(t), \dot{x}(t), y(t), \dot{y}(t))\right\} d t
\end{aligned}
$$

Combining (17) and (18), we have

$\int_{a}^{b} \lambda^{T} f(t, x(t), \dot{x}(t), y(t), \dot{y}(t)) d t \geqq \int_{a}^{b} \lambda^{T} f(t, u(t), \dot{u}(t), v(t), \dot{v}(t)) d t$
Hence

$\int_{a}^{b} f(t, x(t), \dot{x}(t), y(t), \dot{y}(t)) d t \leq \int_{a}^{b} f(t, u(t), \dot{u}(t), v(t), \dot{v}(t)) d t$

For the validation of strong and converse duality theorems we will need to fix $\lambda=\bar{\lambda}$ in the problems (VP) and (VD) respectively and the corresponding problems will be denoted by $(V P)_{\bar{\lambda}}$ and $(V D)_{\bar{\lambda}}$.

Theorem 2. (Strong duality). Assume that the hypotheses of Theorem 1 are satisfied.

Let

$\left(\mathrm{C}_{1}\right):(\lambda, x(t), y(t), \mu(t))$ be properly efficient for $(V P)_{\lambda}$

$$
\begin{aligned}
& \left(\mathrm{C}_{2}\right): \int_{a}^{b}\left[\begin{array}{c}
\psi_{1}(t)^{T}\left\{\begin{array}{c}
\lambda^{T} f_{y y}-\mu(t)^{T} q_{y y} \\
D\left(\lambda^{T} f_{\dot{y} y}-\mu(t)^{T} q_{\dot{y} y}\right.
\end{array}\right\} \\
+D\left\{\psi_{1}(t)^{T} D\left(\lambda^{T} f_{\dot{y} \dot{y}}-\mu(t)^{T} q_{\dot{y} y}\right)\right\} \\
D^{2}\left\{\psi_{1}(t)^{T}\left(\lambda^{T} f_{\dot{y} \dot{y}}-\mu(t)^{T} q_{\dot{y} \dot{y}}\right\}\right.
\end{array}\right] \Psi_{1}(t) d t=0 \\
& \Rightarrow \psi_{1}(t)=0, t \in I \\
& \left(\mathrm{C}_{3}\right):\left\{\begin{array}{l}
\left(f_{y}^{1}-D f_{\dot{y}}^{1}\right),\left(f_{y}^{2}-D f_{\dot{y}}^{2}\right), \ldots \ldots \ldots,\left(f_{y}^{p}-D f_{\dot{y}}^{p}\right) ; \\
\left(\mu(t)^{T} q_{y}-D \mu(t)^{T} q_{\dot{y}}\right)
\end{array}\right\}
\end{aligned}
$$

be linearly independent.

$\left(\mathrm{C}_{4}\right): \rho(t)^{T} p_{x}-D \rho(t)^{T} p_{\dot{x}} \leq 0, \rho(t) \geq 0 \Rightarrow \rho(t)=0, t \in I$ and

$\left(\mathrm{C}_{5}\right): \mu(a)=0=\mu(b)$.

Then there exists $\gamma(t) \in R^{p}, t \in I$ such that $(\lambda, x(t), y(t), \gamma(t))$ is properly efficient for $(V D)_{\bar{\lambda}}$.

Proof: Since $(\lambda, x(t), y(t), \mu(t))$ is a properly efficient solution, it is also a weak minimum. Hence, applying the results of Craven [14] along with the analysis in [15], there exist $\alpha \in R^{p}, r \in R, \eta \in R^{p}, z \in R$ and piecewise smooth $\theta: I \rightarrow R^{n}, \delta: I \rightarrow R^{k}$ and $\beta: I \rightarrow R^{l}$ such that

$$
\begin{aligned}
& H=\alpha f+(\beta(t)-r y(t))^{T}\left[\left(\lambda^{T} f_{y}-\mu(t)^{T} q_{y}\right)-D\left(\lambda^{T} f_{\dot{y}}-\mu(t)^{T} q_{\dot{y}}\right)\right] \\
& -z \mu(t) q-\delta(t)^{T} p(t)-\theta(t)^{T} x(t)-\beta(t)^{T} \mu(t)-\eta^{T} \lambda
\end{aligned}
$$

\section{Satisfying}

$$
\begin{aligned}
& H_{x}-D H_{\dot{x}}+D^{2} H_{\ddot{x}}=0, t \in I \\
& H_{y}-D H_{\dot{y}}+D^{2} H_{\ddot{y}}=0, t \in I \\
& H_{\mu}-D H_{\dot{\mu}}=0, t \in I \\
& H_{\lambda}=0, t \in I
\end{aligned}
$$




$$
\begin{aligned}
& \beta(t)^{T}\left[\left(\lambda^{T} f_{y}-\mu(t)^{T} q_{y}\right)-D\left(\lambda f_{\dot{y}}-D \mu(t)^{T} q_{\dot{y}}\right)\right]=0, t \in I \\
& r \int_{a}^{b} y(t)^{T}\left[\left(\lambda^{T} f_{y}-\mu(t)^{T} q_{y}\right)-D\left(\lambda f_{\dot{y}}-D \mu(t)^{T} q_{\dot{y}}\right)\right]=0, t \in I \\
& \delta(t)^{T} p(t, x, \dot{x})=0, t \in I \\
& z \int_{a}^{b} \mu(t)^{T} q d t=0 \\
& \theta(t)^{T} x(t)=0, t \in I \\
& \beta(t)^{T} \mu(t)=0, t \in I \\
& \eta^{T} \lambda=0 \\
& (\alpha, \beta(t), r, z, \delta(t), \eta, \theta(t)) \geq 0, t \in I \\
& (\alpha, \beta(t), r, z, \delta(t), \eta, \theta(t)) \neq 0, t \in I
\end{aligned}
$$

The relation (19) gives,

$\alpha\left(f_{x}-D f_{\dot{x}}\right)+(\beta(t)-r y(t))^{T}\left(\lambda^{T} f_{y x}-D \lambda^{T} f_{\dot{y} x}\right)$

$+D\left[(\beta(t)-r y(t))^{T}\left(\lambda^{T} f_{y \dot{x}}-D \lambda^{T} f_{\dot{y x}}-\lambda^{T} f_{j x}\right)\right]$

$-D^{2}\left[(\beta(t)-r y(t))^{T}\left(\lambda^{T} f_{\dot{y} \dot{x}}\right)\right]$

$-\left(\delta(t)^{T} p_{x}-D \delta(t) p_{\dot{x}}\right)=\theta(t), t \in I$

The relation (20) implies,

$$
\begin{aligned}
& (\alpha-r \lambda)^{T}\left(f_{y}-D f_{\dot{y}}\right)+(r-z)\left(\mu(t)^{T} q_{y}-D \mu(t)^{T} q_{y}\right) \\
& +(\beta(t)-r y(t))^{T}\left\{\begin{array}{l}
\left(\lambda^{T} f_{y y}-\mu(t)^{T} q_{y y}\right)- \\
D\left(\lambda^{T} f_{\dot{y y}}-\mu(t)^{T} q_{\dot{y y}}\right)
\end{array}\right\} \\
& +D\left[(\beta(t)-r y(t))^{T} D\left(\lambda^{T} f_{\dot{y y}}-\mu(t)^{T} q_{\dot{y y}}\right)\right]+ \\
& D^{2}\left[(\beta(t)-r y(t))^{T}\left(\lambda^{T} f_{\dot{y y}}-\mu(t)^{T} q_{\dot{y y}}\right)\right]=0, t \in I
\end{aligned}
$$

From (21), we have

$$
\begin{aligned}
& -(\beta(t)-r y(t))^{T} q_{y}+(\beta(t)-r y(t))^{T} D q_{\dot{y}}- \\
& D(\beta(t)-r y(t)) q_{\dot{y}}-z q-\beta(t)^{T}=0
\end{aligned}
$$

From (22), we have

$$
(\beta(t)-r y(t))^{T}\left(f_{y}-D f_{\dot{y}}\right)-\eta=0
$$

Since $\eta \geqq 0$ and $\lambda>0$, the equation (20) implies $\eta=0$. Consequently, (33) implies

$(\beta(t)-r y(t))^{T}\left(f_{y}-D f_{\dot{y}}\right)=0$

Multiplying (34) by $\mu(t)^{T}$ then integrating along with using (26) and (28), we have

$$
0=\int_{a}^{b}\left[\begin{array}{l}
-(\beta(t)-r y(t))^{T}\left(\mu(t)^{T} q_{y}\right)+(\beta(t)-r y(t))^{T} \mu(t)^{T} D q_{\dot{y}}- \\
\mu(t)^{T} D(\beta(t)-r y(t))^{T} q_{\dot{y}}
\end{array}\right] d t
$$

This, on using $\mu(t)^{T} D q_{\dot{y}}=D \mu(t)^{T} D q_{\dot{y}}-\dot{\mu}(t)^{T} D q_{\dot{y}}$ gives

$$
\begin{aligned}
& 0=\int_{a}^{b}\left[\begin{array}{l}
-(\beta(t)-r y(t))^{T}\left(\mu(t)^{T} q_{y}\right)+(\beta(t)-r y(t))^{T} \\
\left(D \mu(t)^{T} q_{\dot{y}}-\dot{\mu}(t) q_{\dot{y}}\right) \\
-\mu(t)^{T} D(\beta(t)-r y(t))^{T} q_{\dot{y}}
\end{array}\right] d t \\
& 0=\int_{a}^{b}\left[\begin{array}{l}
-(\beta(t)-r y(t))^{T}\left(\mu(t)^{T} q_{y}-D \mu(t)^{T} q_{\dot{y}}\right)- \\
(\beta(t)-r y(t))^{T}\left(\dot{\mu}(t) q_{\dot{y}}\right) \\
-\mu(t)^{T} D(\beta(t)-r y(t))^{T} q_{\dot{y}}
\end{array}\right] d t \\
& =\int_{a}^{b}\left[(\beta(t)-r y(t))^{T}\left(\mu(t)^{T} q_{y}-D \mu(t)^{T} q_{\dot{y}}\right)\right] d t- \\
& \left.\mu(t)^{T}(\beta(t)-r y(t))^{T} q_{\dot{y}}\right|_{t=a} ^{t=b} \\
& +\int_{a}^{b} \mu(t)^{T} D(\beta(t)-r y(t)) d t \\
& -\int_{a}^{b} \mu(t)^{T} D(\beta(t)-r y(t)) d t
\end{aligned}
$$

(By integrating by parts)

Using $\mu(a)=0=\mu(b)$, we have

$$
\int_{a}^{b}\left[(\beta(t)-r y(t))^{T}\left(\mu(t)^{T} q_{y}-D \mu(t)^{T} q_{\dot{y}}\right)\right] d t=0
$$

Multiplying (33) by $(\beta(t)-r y(t))$ and using (36) and (37), we have

$$
\int_{a}^{b}\left[\begin{array}{l}
(\beta(t)-r y(t))^{T}\left\{\begin{array}{l}
\left(\lambda^{T} f_{y y}-\mu(t)^{T} q_{y y}\right)- \\
D\left(\lambda^{T} f_{y y}-\mu(t)^{T} q_{y y}\right)
\end{array}\right\} \\
+D\left[\begin{array}{l}
(\beta(t)-r y(t))^{T} \\
D\left(\lambda^{T} f_{y \dot{y}}-\mu(t)^{T} q_{y y}\right)
\end{array}\right] \\
+D^{2}\left\{\begin{array}{l}
(\beta(t)-r y(t))^{T} \\
D\left(\lambda^{T} f_{y \dot{y}}-\mu(t)^{T} q_{y \dot{y}}\right.
\end{array}\right\}
\end{array}\right](\beta(t)-r y(t)) d t=0
$$

Which, because of the hypothesis $\left(\mathrm{C}_{2}\right)$ gives

$\beta(t)-r y(t)=0, t \in I$

Using (38) in (33), we have

$(\alpha-r \lambda)^{T}\left(f_{y}-D f_{\dot{y}}\right)+(r-z)\left(\mu(t)^{T} q_{y}-D \mu(t)^{T} q_{y}\right)=0$

This, because of linear independence hypothesis, yields $(\alpha-r \lambda)=0, r=z$

Assume that $r=0$. Then (39) and (38) imply $\alpha=0=z$ and $\beta(t)=0, t \in I$

The relation (32) implies

$\left(\delta(t)^{T} p_{x}-D \delta(t)^{T} p_{\dot{x}}\right)=-\theta(t) \leqq 0, \delta(t) \geqq 0, t \in I$

which because of hypotheses $\left(\mathrm{C}_{4}\right)$ gives 
$\delta(t)=0, t \in I$

Consequently $(\alpha, \beta(t), \eta, \theta(t), z)=0$, contradicting $(31)$

Hence $\alpha>0, z>0 \Rightarrow \alpha=r \lambda>0$.

$\alpha\left(f_{x}-D f_{\dot{x}}\right)-\delta(t)^{T} p_{x}+D \delta(t)^{T} p_{\dot{x}}=\theta(t), t \in I$

$r \lambda\left(f_{x}-D f_{\dot{x}}\right)-\delta(t)^{T} p_{x}+D \delta(t) p_{\dot{x}}=\theta(t), t \in I$

$\Rightarrow\left(\lambda^{T} f_{x}-\frac{\delta(t)}{r} p_{x}\right)-D\left(\lambda^{T} f_{\dot{x}}-\frac{\delta(t)}{r} p_{\dot{x}}\right) \geq 0, t \in I$

and

$\Rightarrow x(t)^{T}\left[\left(\lambda^{T} f_{x}-\frac{\delta(t)}{r} p_{x}\right)-D\left(\lambda^{T} f_{\dot{x}}-\frac{\delta(t)}{r} p_{\dot{x}}\right)\right]=0, t \in I$

from (14)

$q \leq 0, t \in I$

$\beta(t)-r y(t)=0$

$\beta(t)=\frac{\delta(t)}{r} \geq 0$

$\delta(t)^{T} p=0$

From (40), (41), (42) and (43), it follows that $\left(x, \lambda, y, \frac{\delta(t)}{r}\right)$ is feasible for the dual and the objective functional are equal there.

If $\left(\bar{\lambda}, \bar{x}, \bar{y}, \frac{\bar{\delta}(t)}{r}\right)$ is not efficient for (VD) with $\lambda=\bar{\lambda}$, then there exists a point $\left(\bar{\lambda}, \bar{u}, \bar{v}, \frac{\bar{\delta}(t)}{r}\right)$ such that

$\int_{a}^{b} f(t, \hat{u}, \dot{\hat{u}}, \widehat{v}, \dot{\bar{v}}) d t \geq \int_{a}^{b} f(t, \bar{x}, \dot{\bar{x}}, \bar{y}, \dot{\bar{y}}) d t$

which contradicts the conclusion of Theorem 1. Now, it remains to show that $\left(\bar{\lambda}, \bar{x}, \bar{y}, \frac{\bar{\delta}(t)}{r}\right)$ is properly efficient. If it is not so, then for some $\left(\bar{\lambda}, \bar{u}, \bar{v}, \frac{\bar{\delta}(t)}{r}\right) \in G$ and some $i$,

$\int_{a}^{b} f^{i}(t, \hat{u}, \dot{\hat{u}}, \widehat{v}, \dot{\bar{v}}) d t>\int_{a}^{b} f^{i}(t, \bar{x}, \dot{\bar{x}}, \bar{y}, \dot{\bar{y}}) d t$

and

$\left[\int_{a}^{b}\left\{f^{i}(t, \hat{u}, \dot{\vec{u}}, \hat{v}, \dot{\bar{v}}) d t-\int_{a}^{b} f^{i}(t, \bar{x}, \dot{\bar{x}}, \bar{y}, \dot{\bar{y}})\right\} d t\right]>$

$M\left[\int_{a}^{b}\left\{f^{j}(t, \bar{x}, \dot{\bar{x}}, \bar{y}, \dot{\bar{y}}) d t-\int_{a}^{b} f^{j}(t, \hat{u}, \dot{\bar{u}}, \hat{v}, \dot{\bar{v}}) d t\right\}\right]$

For any $M>0$ and all $\mathrm{j}$ satisfying

$\int_{a}^{b} f^{j}(t, \bar{x}, \dot{\bar{x}}, \bar{y}, \dot{\bar{y}}) d t>\int_{a}^{b} f^{j}(t, \hat{u}, \dot{\bar{u}}, \hat{v}, \dot{\bar{v}}) d t$.
This means that

$\left[\int_{a}^{b}\left\{f^{i}(t, \widehat{u}, \dot{\bar{u}}, \widehat{v}, \dot{\bar{v}})-f^{i}(t, \bar{x}, \dot{\bar{x}}, \bar{y}, \dot{\bar{y}})\right\} d t\right]$

can be made arbitrary large whereas

$\left[\int_{a}^{b}\left\{f^{j}(t, \bar{x}, \dot{\bar{x}}, \bar{y}, \dot{\bar{y}})-f^{j}(t, \widehat{u}, \dot{\hat{u}}, \widehat{v}, \dot{\bar{v}})\right\} d t\right]$

Is finite for all $j \neq i$ Since $\bar{\lambda}=\left(\bar{\lambda}^{1}, \bar{\lambda}^{2} \ldots \ldots . . \bar{\lambda}^{p}\right)>0$, we get

$\bar{\lambda}^{i}\left[\int_{a}^{b}\left\{f^{i}(t, \hat{u}, \dot{\bar{u}}, \hat{v}, \dot{\bar{v}})-f^{i}(t, \bar{x}, \dot{\bar{x}}, \bar{y}, \dot{\bar{y}})\right\} d t\right]>$

$\sum_{j \neq i} \bar{\lambda}^{j}\left[\int_{a}^{b}\left\{f^{j}(t, \bar{x}, \dot{\bar{x}}, \bar{y}, \dot{\bar{y}})-f^{j}(t, \hat{u}, \dot{\bar{u}}, \hat{v}, \dot{\bar{v}})\right\} d t\right]$

or

$\int_{a}^{b} \bar{\lambda}^{T} f(t, \hat{u}, \dot{\hat{u}}, \hat{v}, \dot{\hat{v}}) d t>\int_{a}^{b} \bar{\lambda}^{T} f(t, \bar{x}, \dot{\bar{x}}, \bar{y}, \dot{\bar{y}}) d t$.

This contradicts Theorem 1 and hence theorem is completely established.

A converse duality theorem whose proof is be similar to that of Theorem 2 is merely stated below:

Theorem 3 (Converse duality) : Assume that the hypotheses of Theorem 1 hold.

$\left(\mathrm{H}_{1}\right):(\lambda, x(t), y(t), \gamma(t))$ be a properly efficient solution of $(V D)_{\bar{\lambda}}$.

$\left(\mathrm{H}_{2}\right): \int_{a}^{b}\left[\begin{array}{c}\Psi_{2}(t)^{T}\left\{\begin{array}{l}\lambda^{T} f_{x x}-\gamma(t)^{T} p_{x x}- \\ D\left(\lambda^{T} f_{\dot{x} x}-\gamma(t)^{T} p_{\dot{x} x}\right)\end{array}\right\} \\ -D\left\{\Psi_{2}(t)^{T} D\left(f_{\dot{x} \dot{x}}-\gamma(t)^{T} p_{\dot{x} \dot{x}}\right)\right\} \\ D^{2}\left\{\Psi_{2}(t)^{T} D\left(f_{\dot{x} \dot{x}}-\gamma(t)^{T} p_{\dot{x} \dot{x}}\right)\right\}\end{array}\right]+\Psi_{2}(t) d t=0$.

$\Rightarrow \psi_{2}(t)=0, t \in I$.

$\left(\mathrm{H}_{3}\right)$ :

$\left\{\left(f_{x}^{1}-D f_{\dot{x}}^{1}\right),\left(f_{x}^{2}-D f_{\dot{x}}^{2}\right), \ldots \ldots \ldots,\left(f_{x}^{p}-D f_{\dot{x}}^{p}\right) ;\left(\gamma(t)^{T} p_{x}-D \gamma(t)^{T} p_{\dot{x}}\right)\right\}$ be linearly independent,

$\left(\mathrm{H}_{4}\right): \gamma(a)=0=\gamma(b)$.

$\left(\mathrm{H}_{5}\right): \varsigma(t)^{T} q_{y}-D \varsigma(t)^{T} q_{\dot{y}} \leqq 0$

and $\varsigma(t) \geq 0 \Rightarrow \varsigma(t)=0, t \in I$

Then there exists $\mu(t) \in R^{l}, t \in I \quad$ such that $(\lambda, \bar{x}(t), y(t), \bar{\mu}(t))$ is properly efficient for $(V P)_{\bar{\lambda}}$.

\section{SELF DUALITY}

A mathematical programming problem is said to be self dual, if it is formally identical with its dual, i.e. if the dual is recast in the form of primal, the new problem obtained is the same as the primal. In general the problems (VP) and (VD) are not self dual without an additional condition. 
Suppose that $x(t)$ and $y(t)$ are of the same dimension. The vector function $(t, x(t), \dot{x}(t), y(t), \dot{y}(t))$ is said to be skew symmetric if

$f(t, x(t), \dot{x}(t), y(t), \dot{y}(t))=-f(t, y(t), \dot{y}(t), x(t), \dot{x}(t))$,

for all $\mathrm{x}$ and $\mathrm{y}$ in the domain of $\mathrm{f}$. That is,

$f^{i}(t, x(t), \dot{x}(t), y(t), \dot{y}(t))=-f^{i}(t, y(t), \dot{y}(t), x(t), \dot{x}(t)), i=1,2, \ldots, p$.

It is remarked here that the study of self duality for the problems of preceding section is significant in the sense that a pair of multiobjective symmetric selfdual multiobjective variational problem represent a symmetric vector-valued dynamic game.

Theorem 4. (Self duality). Let

(i) f be skew-symmetric,

(ii) $\quad p(t, x(t), \dot{x}(t))+q(t, y(t), \dot{y}(t))=0, t \in I$

(iii) $\quad p_{x}(t, x(t), \dot{x}(t))+q_{y}(t, y(t), \dot{y}(t))=0, t \in I$

$p_{\dot{x}}(t, x(t), \dot{x}(t))+q_{\dot{y}}(t, y(t), \dot{y}(t))=0, t \in I$, and

(iv) $\quad \mu(t)=\gamma(t), t \in I$

Then (VP) is self dual. Also if (VP) and (VD) are dual variational problem and $(\bar{x}, \bar{y}, \lambda, \mu)$ is a joint properly efficient solution of the problem (VD), then so is $(\bar{y}, \bar{x}, \lambda, \gamma)$ and the common objective functional value is zero, i.e.,

$\int_{a}^{b} f^{i}(t, x(t), \dot{x}(t), y(t), \dot{y}(t)) d t=0, i \in\{1,2, \ldots, p\}$

Proof: The problem (VD) may be re-written as minimization problem:

Minimize

$\left(-\int_{a}^{b} f^{1}(t, u(t), \dot{u}(t), v(t), \dot{v}(t)) d t, \ldots .,-\int_{a}^{b} f^{p}(t, u(t), \dot{u}(t), v(t), \dot{v}(t)) d t\right)$ subject to

$u(a)=0=u(b)$,

$v(a)=0=v(b)$.

$-\lambda^{T} f_{u}(t, u(t), \dot{u}(t), v(t), \dot{v}(t))+\gamma(t)^{T} p_{u}(t, u, \dot{u})$

$\leqq D\left(-\lambda^{T} f_{\dot{u}}(t, u(t), \dot{u}(t), v(t), \dot{v}(t))+\gamma(t)^{T} p_{\dot{u}}(t, u, \dot{u})\right), t \in I$

$\int_{a}^{b} u(t)^{T}\left[\begin{array}{l}\left(\begin{array}{l}-\lambda^{T} f_{u}(t, u(t), \dot{u}(t), v(t), \dot{v}(t)) \\ +\gamma(t)^{T} p_{u}(t, u, \dot{u})\end{array}\right) \\ -D\left(\begin{array}{l}-\lambda^{T} f_{\dot{u}}(t, u(t), \dot{u}(t), v(t), \dot{v}(t))+ \\ \gamma(t)^{T} p_{\dot{u}}(t, u, \dot{u})\end{array}\right)\end{array}\right] d t \geqq 0$.

$-\int_{a}^{b} \gamma(t)^{T} p(t, u(t), \dot{u}(t)) d t \geqq 0$

$-q(t, v(t), \dot{v}(t)) \geqq 0, t \in I$ $v(t) \geqq 0, \gamma(t) \geqq 0, t \in I$

$\lambda>0$.

with skew symmetry of f, we have for $t \in I$,

$\lambda^{T} f_{u}(t, u(t), \dot{u}(t), v(t), \dot{v}(t))=-\lambda^{T} f_{v}(t, v(t), \dot{v}(t), u(t), \dot{u}(t))$

$\lambda^{T} f_{v}(t, u(t), \dot{u}(t), v(t), \dot{v}(t))=-\lambda^{T} f_{u}(t, v(t), \dot{v}(t), u(t), \dot{u}(t))$

$\lambda^{T} f_{\dot{u}}(t, u(t), \dot{u}(t), v(t), \dot{v}(t))=-\lambda^{T} f_{\dot{v}}(t, v(t), \dot{v}(t), u(t), \dot{u}(t))$

In view of these relations along with the hypotheses (i) and (ii), the above minimization problem takes the following form:

Minimize

$\left(\int_{a}^{b} f^{1}(t, v(t), \dot{v}(t), u(t), \dot{u}(t)) d t, \ldots ., \int_{a}^{b} f^{p}(t, v(t), \dot{v}(t), u(t), \dot{u}(t)) d t\right)$

subject to

$u(a)=0=u(b)$
$v(a)=0=v(b)$
$\lambda^{T} f_{v}(t, v(t), \dot{v}(t), u(t), \dot{u}(t))-\mu(t)^{T} q_{v}(t, v(t), \dot{v}(t))$
$\leq D\left(\lambda^{T} f_{\dot{v}}(t, v(t), \dot{v}(t), u(t), \dot{u}(t))-\mu(t)^{T} q_{\dot{v}}(t, v(t), \dot{v}(t))\right), t \in I$

$\int_{a}^{b} u(t)^{T}\left[\begin{array}{l}\left(\begin{array}{l}\lambda^{T} f_{v}(t, v(t), \dot{v}(t), u(t), \dot{u}(t))- \\ \mu(t)^{T} q_{v}(t, v(t), \dot{v}(t))\end{array}\right) \\ -D\left(\begin{array}{l}\lambda^{T} f_{\dot{v}}(t, v(t), \dot{v}(t), u(t), \dot{u}(t))- \\ \mu(t)^{T} q_{\dot{v}}(t, v(t), \dot{v}(t))\end{array}\right)\end{array}\right] d t \geqq 0$.

$\int_{a}^{b} \mu(t)^{T} q(t, v(t), \dot{v}(t)) d t \geqq 0$

$p(t, u(t), \dot{u}(t)) \geqq 0, t \in I$

$v(t) \geqq 0, \mu(t) \geqq 0, t \in I$

$\lambda>0$.

which is just the problem (VP). Hence (VP) is self dual.

Since $(\bar{u}, \bar{v}, \bar{\lambda}, \bar{\mu})$ is properly efficient to the problem (VP) and (VD), the objective functional value are equal to $\int_{a}^{b} f(t, \bar{u}(t), \dot{\bar{u}}(t), \bar{v}(t), \dot{\bar{v}}(t)) d t$

From symmetric duality, the proper efficiency for (VP) implies the proper efficiency of $(\bar{v}, \bar{u}, \bar{\lambda}, \bar{\mu})$ for (VD). By similar arguments, $(\bar{v}, \bar{u}, \bar{\lambda}, \bar{\mu})$ is the solution for (VP). Also, the two objective values are equal to

$\int_{a}^{b} f(t, \bar{v}(t), \dot{\bar{v}}(t), \bar{u}(t), \dot{\bar{u}}(t)) d t$ but

$\int_{a}^{b} f(t, \bar{v}(t), \dot{\bar{v}}(t), \bar{u}(t), \dot{\bar{u}}(t)) d t=$

$-\int_{a}^{b} f(t, \bar{u}(t), \dot{\bar{u}}(t), \bar{v}(t), \dot{\bar{v}}(t)) d t$

By skew symmetry of $\mathrm{f}$ 


$$
\begin{aligned}
& \int_{a}^{b} f(t, \bar{u}(t), \dot{\bar{u}}(t), \bar{v}(t), \dot{\bar{v}}(t)) d t= \\
& -\int_{a}^{b} f(t, \bar{u}(t), \dot{\bar{u}}(t), \bar{v}(t), \dot{\bar{v}}(t)) d t
\end{aligned}
$$

This implies

$$
\int_{a}^{b} f^{i}(t, \bar{u}(t), \dot{\bar{u}}(t), \bar{v}(t), \dot{\bar{v}}(t)) d t=0, i \in\{1,2 \ldots, p\} .
$$

This accomplishes the proof of the theorem.

\section{PROBLEMS WITH NATURAL BOUNDARY VALUES}

It is possible to formulate a pair symmetric dual multiobjective variational problem with natural boundary values rather than fixed end points.

$\left(\mathrm{VP}_{0}\right)$ : Minimize $\int_{a}^{b} f(t, x(t), \dot{x}(t), y(t), \dot{y}(t)) d t$

subject to

$\lambda^{T} f_{y}(t, x(t), \dot{x}(t), y(t), \dot{y}(t))-$

$\mu(t)^{T} q_{y}(t, y(t), \dot{y}(t))$

$-D\left(\begin{array}{l}\lambda^{T} f_{\dot{y}}(t, x(t), \dot{x}(t), y(t), \dot{y}(t))- \\ \mu(t)^{T} q_{\dot{y}}(t, x(t), \dot{x}(t), y(t), \dot{y}(t))\end{array}\right) \leqq 0, t \in I$

$\int_{a}^{b} y(t)^{T}\left[\begin{array}{l}\lambda^{T} f_{y}(t, x(t), \dot{x}(t), y(t), \dot{y}(t))- \\ \mu(t)^{T} q_{y}(t, y(t), \dot{y}(t)) \\ -D\left(\begin{array}{l}\lambda^{T} f_{\dot{y}}(t, x(t), \dot{x}(t), y(t), \dot{y}(t))- \\ \mu(t)^{T} q_{\dot{y}}(t, x(t), \dot{x}(t), y(t), \dot{y}(t))\end{array}\right)\end{array}\right] d t \geqq 0$

$\int_{a}^{b} \mu(t)^{T} q(t, y(t), \dot{y}(t)) \geqq 0, t \in I$

$\lambda>0, x(t) \geqq 0, \mu(t) \geqq 0, p(t, x(t), \dot{x}(t)) \geqq 0, t \in I$

$\lambda^{T} f_{\dot{y}}(t, x(t), \dot{x}(t), y(t), \dot{y}(t))-\left.\mu(t)^{T} q_{\dot{y}}(t, y(t), \dot{y}(t))\right|_{t=a}=0$

$\lambda^{T} f_{\dot{y}}(t, x(t), \dot{x}(t), y(t), \dot{y}(t))-\left.\mu(t)^{T} q_{\dot{y}}(t, y(t), \dot{y}(t))\right|_{t=b}=0$

$\left(\mathrm{VD}_{0}\right)$ : Maximize $\int_{a}^{b} f(t, u(t), \dot{u}(t), v(t), \dot{v}(t)) d t$

subject to

$\lambda^{T} f_{u}(t, u(t), \dot{u}(t), v(t), \dot{v}(t))-\gamma(t)^{T} p_{u}(t, u(t), \dot{u}(t))$

$-D\left(\lambda^{T} f_{\dot{u}}(t, u(t), \dot{u}(t), v(t), \dot{v}(t))-\gamma(t)^{T} p_{\dot{u}}(t, u(t), \dot{u}(t))\right) \geqq 0, t \in I$

$\int_{a}^{b} u(t)^{T}\left[\begin{array}{l}\lambda^{T} f_{u}(t, u(t), \dot{u}(t), v(t), \dot{v}(t))-\gamma(t)^{T} p_{u}(t, u(t), \dot{u}(t)) \\ -D\left(\lambda^{T} f_{\dot{u}}(t, u(t), \dot{u}(t), v(t), \dot{v}(t))-\gamma(t)^{T} p_{\dot{u}}(t, u(t), \dot{u}(t))\right)\end{array}\right] d t \leqq 0$

$\int_{a}^{b} \gamma(t)^{T} p(t, u(t), \dot{u}(t)) d t \leqq 0$. $\lambda>0, v(t) \geqq 0, \gamma(t) \geqq 0, q(t, v(t), \dot{v}(t)) \leqq 0, t \in I$

$\lambda^{T} f_{\dot{u}}(t, u(t), \dot{u}(t), v(t), \dot{v}(t))-\left.\gamma(t)^{T} p_{\dot{u}}(t, u(t), \dot{u}(t))\right|_{t=a}=0$

$\lambda^{T} f_{\dot{u}}(t, u(t), \dot{u}(t), v(t), \dot{v}(t))-\left.\gamma(t)^{T} p_{\dot{u}}(t, u(t), \dot{u}(t))\right|_{t=b}=0$

\section{NONLINEAR MULTIOBJECTIVE PROGRAMMING PROBLEMS}

If all the functions in the problem $\left(\mathrm{VP}_{0}\right)$ and $\left(\mathrm{VD}_{0}\right)$ are independent of $t$, then the problems reduce to the following problem treated by Chandra and Prasad [11]

$\left(N P_{1}\right)$ Minimize $f(x, y)$

subject to $\lambda^{T} f_{y}(x, y)-\mu^{T} q_{y}(y) \leqq 0$

$y^{T}\left(\lambda^{T} f_{y}(x, y)-\mu^{T} q_{y}(y)\right) \geqq 0$

$\mu^{T} q(y) \geqq 0$

$\lambda>0, x \geqq 0, \mu \geqq 0, p(x) \geqq 0$

$\left(N P_{2}\right)$ : Maximize $f(u, v)$

subject to $\lambda^{T} f_{u}(u, v)-\gamma^{T} p_{u}(u) \geqq 0$

$u^{T}\left(\lambda^{T} f_{u}(u, v)-\gamma^{T} p_{u}(u)\right) \leqq 0$

$\gamma^{T} p(u) \leqq 0$

$\lambda>0, v \geqq 0, \gamma(t) \geqq 0, q(v) \leqq 0$.

\section{CONCLUSION}

In this research equivalence between a certain Constraints vector-valued dynamic game and a pair of multiobjective symmetric dual variational problem is established. A number of duality results for this pair of variational problems are derived under appropriate generalized convexity assumptions. Selfduality reflecting symmetric dynamic games is studied. The linkage between our results and those of the static case is pointed out. The results validated in this research can be revisited in the context of optimal control.

\section{CONFLICT OF INTEREST}

The authors confirm that this article content has no conflict of interest.

\section{ACKNOWLEDGEMENTS}

The authors are grateful to an anonymous referee for his is valuable comments which have improved the presentation of this research paper.

\section{REFERENCES}

[1] Charnes A. Constrained games and linear programming. Proc Natl Acad Sci USA 1953; 39: 639-41.

[2] Cottle RW. An infinite game with a convex-concave pay-off kernel. Research report no. ORC 63-19 (RN-2). Operations Research Centre, University of California, Berkeley 1963. 
[3] Chandra S, Mond B, Smart I. Constrained games and symmetric duality with pseudo- invexity. Opsearch 1919; 27(1): 14-30.

[4] Corley HW. Games with vector pay-offs. J Opt Theory Appl 1976; 47: 491-8.

[5] Durga Prasad MV, Sreenivas PC. Vector-valued non-zero sum games and non-linear programming. Opsearch 1997; 34(3): 180-5.

[6] Mond B, Chandra S, Durga Prasad MV. Constrained games and symmetric duality. Opsearch 1987; 24: 69-77.

[7] Kawaguchi T, Maruyama V. A note on mini-max (Max-min) programming. Manag Sci 1976; 22: 670-6.

[8] Mond B, Weir T. Generalized concavity and duality. In: Schaible S, Ziemba WT, Eds. Generalized concavity in optimization and economics. Academic Press: New York 1981; pp. 263-79.
[9] Husain I, Ahmad B. Constrained dynamic game and symmetric duality for variational problems. J Math Sys Sci 2012; 2: 171-6.

[10] Mond B, Hanson MA. Symmetric duality for variational problems. J Math Anal Appl 1967; 18: 161-72.

[11] Chandra S, Durga Prasad MV. Constraint vector valued games and multiobjective programming. Opsearch 1992; 19(1): 1-10.

[12] Zhokovskii VI, Salukvadze M. The vectoe-valued maximin. Academic Press: New York 1994.

[13] Bector CR, Husain I. Duality for multiobjective variational problems. J Math Anal Appl 1992; 166: 214-29.

[14] Craven BD. Lagrangian conditions and quasiduality. Bull Aust Math Soc 1977; 16: 325-39.

[15] Bector CR, Chandra S, Husain I. Generalized concavity and duality in continuous programming. Utilitas Math 1984; 25: 171-90.

(C) Husain and Jain; Licensee Bentham Open .

This is an open access article licensed under the terms of the Creative Commons Attribution Non-Commercial License (http://creativecommons.org/licenses/ by-nc/3.0/) which permits unrestricted, non-commercial use, distribution and reproduction in any medium, provided the work is properly cited. 